\title{
Upper gastrointestinal haemorrhage
}

\section{H Lim, D M Chalmers}

\section{Answers on $p 494$.}

A 75 year old man presented with a three day history of melaena without any abdominal pain. He had one episode of haematemesis (about $100 \mathrm{ml}$ blood) in the emergency room. He had no previous history of peptic ulcer disease but had significant heart disease with coronary arterial by pass graft in 1992, poor left ventricular function, paroxysmal atrial fibrillation, and type 2 diabetes. His regular medications included aspirin, warfarin, bisoprolol, bumetamide, amiodarone, lisinopril, and metformin. He was tachycardic with a pulse of 110 beats $/ \mathrm{min}$ and blood pressure of $110 / 66 \mathrm{~mm} \mathrm{Hg}$. No abdominal tenderness was noted on examination and rectal examination revealed black tarry stool.

Laboratory tests showed a haemoglobin of $93 \mathrm{~g} / \mathrm{l}(135-180)$ and international normalised ratio of 3.0. He was initially treated with $5 \mathrm{mg}$ of vitamin $\mathrm{K}$ intravenously, fresh frozen plasma, and blood transfusion. An urgent upper gastrointestinal endoscopy showed excess altered and clotted blood in the fundus of stomach and duodenum but no source of bleeding was seen. He had no further overt bleed overnight but had six units of blood transfusion guided by the haemoglobin level. A repeat upper gastrointestinal endoscopy the following morning showed fresh clotted blood in the fundus of stomach with a bleeding source seen on the body of the stomach (fig l).

\section{QUESTIONS}

(1) What is the diagnosis?

(2) What is the most appropriate endoscopic haemostatic method?

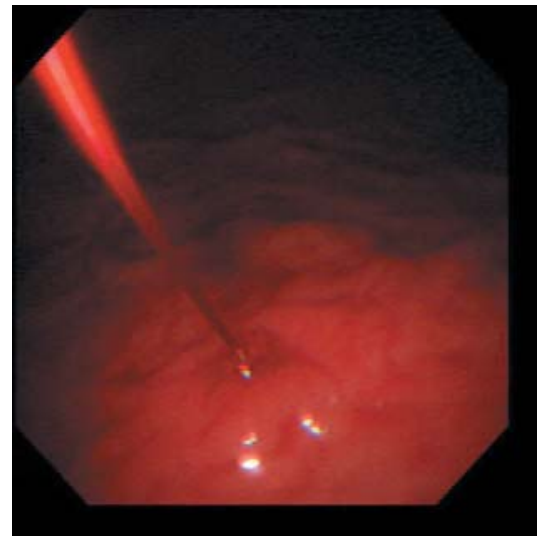

Figure 1 Spurting lesion in stomach.

Postgrad Med J 2004;80:492.

doi: 10.1136/pgmi.2003.016055

$\ldots \ldots \ldots \ldots \ldots \ldots$

\section{Authors' affiliations}

C H Lim, D M Chalmers, Department of Gastroenterology, Leeds General Infirmary, Leeds, UK

Correspondence to: Dr C H Lim, Department of Gastroenterology, Room 190A, Clarendon Wing, The General Infirmary at Leeds, Great George Street, Leeds LSI 3EX, UK; ch.lim@virgin.net

Submitted 14 October 2003 Accepted 15 October 2003 